bei welchen diese erste Lieferung abbricht. Wenn S. 187 bei dem Artikel Phosphor erwähnt ist, dass bei Vergiftungen durch diesen der Tod durch Magen- und Darmentzündung erfolge, und man nach Anwendung von 0 el und Brechmitteln gebrannte Magnesia mit lauem schleimigen Getränke geben solle, so hat sich nach in Dresden an Thieren angestellten Versuchen ergeben, dass der Tod nicht durch Entzündung, sondern durch Zersetzung der Blutmasse erfolgt und Magnesia leider ohne nützliche Wirkung sich erweist.

Bei Vergleichung dieser Bogen der zweiten Auflage mit denen der ersten ergiebt sich, dass allerdings eine Umarbeitung zum Vortheile des Buches statt gefunden hat. Wir hoffen, dass die folgenden Hefte diesem ersteren entsprechen werden, und dürfen alsdann dasselbe als ein sehr brauchbares Lehrbuch der Pharmacie bezeichnen.

Dr. L. F. Bley.

Schilderung der deutschen Pflanzenfamilien vom botanischdescriptiven und physiologisch-chemischen Standpuncte, von Dr. Hermann Hof fmann, Privatdocenten an der Ludwigs-Universität zu Giessen. Verlag von G. F. Hejer zu Giessen. 1846.

Der Verfasser hat, wie er in der Einleitung sagt, versucht, an unsern deutschen Pflanzen zu zeigen, wie weit eine allseitige Betrachtung derselben möglich und erfolgreich sei, und zu beweisen, dass jede Seite des Pflanzenlebens gleiche Berechtigung an unsere Aufmerksamkeit hat, und dass nur bei gleichmässiger Betrachtung aller Seiten ein Erfolg von diesen Studien zu erwarten ist. $\mathrm{Er}$ setzt nun auseinander, wie er das vorgesteckte Ziel zu erreichen hofft, und spricht, indem er zugleich eine Anweisung zum Gebrauche seines Buches geben will, 1) vom System, 2) von der Diagnose der Familien, 3) von der Verwandtschaft nach der Form, 4) von der Literatur, 5) von den Abbildungscitaten, 6) über die deutschen Genera, 7) über die dargebotenen Beispiele, 8) über das, was unter der Ueberschrift Chemie dargeboten werden soll, 9) über den Abschnilt, der Belege zur Chemie geben soll, 10) von dem Vorkommen, 11) über die Belege zum Vorkommen, 12) von der Anwendung, 13) von der Wirkungsweise. Es ist leicht zu erkennen, dass in diese Rubriken Alles aufgenommen werden kann, was zu einer allseitigeren und tieferen Auffassung der Botanik zu führen im Stande ist. Nachdem nun eine Uebersicht des natürlichen Systems nach Sectionen, Classen und Familien, hauptsächlich nach Endlicher, gegeben ist, wird zu der Schilderung der Familien, die nach der oben mitgetheilten Eintheilung behandelt sind, übergegangen. Um das, was hier gewährt ist, beurtheilen zu können, scheint es mir am zweckmassigsten, eine Probe der Behandlung einer Familie aufzuführen; zuvor aber auch noch die Diagnose der Classe mitzutheilen.

$$
\text { Class. 50. Terebinthi. }
$$

(Fig. 144-147.)

Di agnose. Mleist holzige Pflanzen mit balsamischen Saften und ätheriscli-öligen Absonderungen in den Blättern. Blüthen of unvollständig, Kelch meist frei. Krone aus Kelchblältern von gleicher Zahl. Eichen in den Fächern des Fruchtknotens mangeln, zu zweien coder mehren). Staubgefässe meist doppelt so viel als Kronblätter. 
144. Familie. Juglandeae. Wallnussartige. (Fig. 144.)

Diagnose. Blüthen einbäusig. Nännliche Blüthen in Kätzchen, weibliche Blüthen einzeln oder zu zweien bis dreien am Ende der Aestchen, ohne Hülle, mit vier krautigen Blumenblättern. Fruchtknoten einfächerig, eineiig. Eichen aufrecht. Fleischige Steinfrucht (mit zweiklappiger Nussschale). Samen eiveisslos mit faltig gewundenen Keimblattern.

Verwandt mit Burseraceen, Anacardiaceen, Capaliferen (Amentaceen).

Literatur (Krug. pag. 404) Spach in Nouv. Annal. de sc. nat. XV. p. 349 und 359 . - ( 0 ke n tab. 20.)

Genera Germanica. 1183. Juglans, Wallnuss (XXI. 8. Nr. 3. 2.)

Ch e mi e. Ein oft sehr verbreitetes Aroma verbindet diese Familie mit den übrigen dieser Classe, es hat seinen hauptsächlichsten Sitz in den grünen Theilen. Im Holzsaft hat Langlo is Salpetersäure, Aepfelsäure, Milchsäure etc. aufgefunden. Die grüne Fruchthülle der Wallnuss enthält einen scharfen Bitterstoff, welcher an der Luft braungefärbt und abgestumpft wird; daneben vielleicht Gerbsäure, in unreifem Zustande Stärke und Zucker (zum Theil lrystallisirbar nach W ack enroder), gummiarlige Substanzen, Essigsäure (Bernays), Aepfelsäure (Braconnol), Citronensäure und 0xalsäure (nach demselben); in der Asche scheinen die alkalischen Salze zu überwiegen. - Der Samen enthält eine bedeutende Menge 0el, welches im Keime mild, in dem Häutchen von scharfer Beschaffenheit ist. Daneben findet sich ähnlich wie bei vielen öligen Samen, Legumin; Stärke scheint zu fehlen. Mitunter ist hier viel Bitterstoff, so namentlich bei Carya amara.

Beläge. Juglans: B iot. Langlois (Pharm. Centralbl. März 44.). Fechner p. 17. (Braconnot, Wackenroder.) Bernays (Pharm. Centralbl. 1845. No.41) Legumin fanden Cahours und Dumas im Samen, v. Baumhauer (Pharm. Centralbl. 44. p.603.), S ch übler (Agric. Ch. II. 197). Nach P ayen sind die Blätter reich an kohlensaurem Kalk. - Wolff p. 631 (Langlois) p. 687 (Brac.); p. 311 (de Saussüre); p. 317 (de S.); Buchner, Wolff p. 456 (v. Ba $\mathrm{mh}$ a uer).

Vor k ommen. Neist in Nordamerika.

Anwendung. Juglans regia L. (Dss. 4. 2. [96.]; Krebs T. 38.), der gemeine Wallnussbaum aus Persien, liefert die Nuc. Jugl. immat. und Cort. nuc. Jugl. - Die Blätter gegen Skropheln. Auch des schönen Holzes, der öligen und essbaren Samenkerne wegen wird diese Art vielfach gepllanzt. In den Anlagen sieht man ausserdem mehre nordamerikanische Arten, Juglans cinerea, nigra (Black, Hickory), Carya alba, amara (White, Hickory) u. A.

Gewiss wird durch diese wortgetreue Mittheilung eines Artikels am besten dargethan, welcher Werth der Behandlungsweise beizulegen ist; gewiss wird aber auch das so gegebene treue Bild das ganze Buch als ein beachtenswerthes und nützliches erscheinen lassen, welches besonders den Pharmaceuten, die Chemie und Botanik mit gleicher Liebe treiben, sehr willkommen sein wird.

In einem Anhange finden sich noch: 1) Gebirgs- und BodenAnalysen, 2) Formeln der Pflanzenstoffe, 3) Linnés Sexualsystem und 4) eine Erläuterung der Abkürzungen. Ein Register erleichtert 
das Auffinden der einzelnen Familien und Gattungen, und 12 Tafeln Abbildungen dienen zur Erläuterung der diagnostischen Analyse.

$$
\text { Dr. Geiseler. }
$$

\section{Lehrbuch der Chemie von E. Mitscherlich, Geh. Medi- cinalrath etc. Erster Band. Vierte Auflage. Berlin 1847. Mittler.}

Mitscherlich's Lehrbuch der Chemie ist zwar zu bekannt und hat einen zu grossen Werth, als dass auf dasselbe noch besonders hingewiesen werden darf, doch mögen einige Worte über das Buch hier einen Platz finden. Allgemein wird bedauert, dass es noch nicht vollendet ist. Der erste Theil nur ist in der vierten Auflage erschienen, er enthält die Metalloide und in einer zweiten Abtheilung die Säuren. Wer die Vorträge des Verfassers über Chemie gehört hat, der kennt die von ihm gewählte Lehrmethode. Es wird sogleich mit der Anstellung von ersuchen angefangen und aus ihnen werden die chemischen Gesetze und Theorien abgeleitet. In dem vorliegenden Lehrbuche wird ebenso verfahren; an die Beschreibung von Versuchen, die durch Abbildungen noch deullicher gemacht werden, knüpft sich die Entwickelung der wichtigsten allgemeinen Begriffe, und schon in der ersten Abtheilung sind die hauptsächlichsten Thatsachen so gegeben, dass nach Abbandlung der Metalloide und ihrer Verbindungen, über Zusammensetzung der atmosphärischen Luft, über Entwickelung von Wärme und Licht bei der Verbrennung und über die chemische Verwandtschaftslraft, mil Erläuterung des Gesetzes der bestimmten Proportionen und der alomistischen Theorie in einer Weise gesprochen werden kann, die ein vollständiges Verständniss erwarten lässt. Besonders zu erwähnen ist noch, dass in einem unmittelbar auf Kohlenstoff folgenden Abschnitle einige Verbindungen, die aus Kohlenstoff, Wasserstoff, Sauerstoff und Stickstoff bestehen, abgehandelt sind, und zwar in Gruppen zusammengestellt, da, wie es in der Vorrede heisst, dieser Theil der Wissenschaft noch so in der Entwickelung begriffen ist, dass allgemeine Gesichtspuncte zu gewinnen nur für eine gewisse Anzahl von Verbindungen möglich war. In der zweiten Abtheilung des vorliegenden ersten Bandes finden wir die Sauerstoffsäuren und Oxyde der Metalloide, die Säuren mit zusammengesetzlem Radical, die Säuren der Oele und Feltarten, die Säuren, welche statt Sauerstoff Schwefel oder andere Substanzen enthalten, die Säuren des Wasserstoffs und die zusammengesetzten Säuren des Wasserstoffs abgehandelt, und sehen so, wenn auch nicht ein durchaus vollständiges, doch ein in sich geschlossenes und sehr werthyolles Ganze schon in dem ersten Bande von Mitscherlich's Chemie vor uns. Der Fortsetzung wird gewiss von allen Seiten mit freudiger Erwartung entgegengesehen.

Der zweite Band wird, nach der in der Vorrede gegebenen Verheissung, die Metalle und ihre Verbindungen enthaltend, in ciner zweiten Auflage erscheinen, den Schluss des Lehrbuchs aber wird ein dritter Band bilden, der die vegetabilischen Alkalien enthalten wird, ferner einige in dem ersten Bande nicht abgehandelte Gruppen von Kohlenstoff-, Stickstoff-, Wasserstoff- und Sauerstoff-Verbindungen, ein Verzeichniss einzeln stehender, noch nicht gehörig untersuchter Verbindungen, eine kurze Anleitung zur chemischen Analyse, verschiedene 